\title{
RHEUMA AKADEMIE Veranstaltungen der Rheumatologischen Fortbildungsakademie 2018
}

\author{
Wir freuen uns auf Ihre Anmeldung über \\ info@rheumaakademie.de
}

\begin{abstract}
2018
Informationen $z u$ den aktuellen Terminen finden Sie auch unter www.rheumaakademie.de

\section{0.-21.04.2018, Berlin CME: Zertifizierung wird beantragt}

13. Kongress des Berufsverbandes Deutscher Rheumatologen Fachliche Leitung:

Dr. Ludwig Kalthoff

Prof. Dr. Jörn Kekow
\end{abstract}

21.04.2018, Berlin

StruPI-RA (Train-the-Trainer-

Seminar)

Patienteninformation -

Rheumatoide Arthritis

Fachliche Leitung:

Prof. Dr. med. Ina Kötter

Dr. med. Florian Schuch

02.05.2018, Kirchheim unter

Teck

Voneinander lernen - Behandlungskoordination von Patienten mit rheumatologischen Erkrankungen (Train-the-Trainer-Seminar)

Fachliche Leitung:

Prof. Dr. med. Jürgen Braun

Prof. Dr. med. Klaus Krüger

Dr. med. Kirsten Karberg
16.05.2018, Tübingen

Kapillarmikroskopie (Teil 2)

Fachliche Leitung:

Dr. med. Walter Hermann

25.-27.05.2018, Berlin

CME: Zertifizierung wird

beantragt

Weiterbildungswochenende

Rheumatologie

25.05.2018: Rheuma und Alter

26.05.2018: Medizinische Begutachtung in der Rheumatologie (Teil 1)

27.05.2018: Medizinische Begutachtung in der Rheumatologie (Teil 2)

23.-24.06.2018, Köln

Rheumatologische Fachassistenz

Refresh- und Updatekurs

Fachliche Leitung:

Prof. Dr. med. Peter Kern

Dr. med. Stefan Kleinert

Prof. Dr. med. Klaus Krüger

Dr. med. Florian Schuch

27.-30.06.2018, Düsseldorf

CME: Zertifizierung wird

beantragt

Prüfungsvorbereitungskurs

Rheumatologie

Fachliche Leitung:

Prof. Dr. med. Jürgen Braun

Prof. Dr. med. Klaus Krüger

Prof. Dr. med. Bernhard Manger

Prof. Dr. med. Matthias Schneider
04.-07.07.2018, Potsdam

CME: voraussichtlich 22

22. Rheumatologische

Sommerakademie

Fachliche Leitung:

Prof. Dr. med. Ina Kötter,

Dr. med. Johannes Mattar

06.07.2018, Berlin

CME: Zertifizierung wird be-

antragt

Treffpunkt Rheumatologie

Fachliche Leitung:

Prof. Dr. med. Erika

Gromnica-Ihle

01.09.-09.12.2018, Berlin

Rheumatologische Fachassistenz

\section{Grundkurs}

Fachliche Leitung:

Dr. med. Florian Schuch

05.09.-08.09.2018, Haigerloch

CME: voraussichtlich 26

23. Rheumatologische

Sommerakademie

Fachliche Leitung:

Prof. Dr. med. Ina Kötter,

Dr. med. Johannes Mattar

\author{
07.-09.09.2018, Herne \\ CME: Zertifizierung wird \\ beantragt \\ Weiterbildungswochenende \\ Rheumatologie \\ 07.09.2018: Kinderwunsch und \\ Schwangerschaft \\ 08.09.2018: Kapillarmikrosko- \\ pie (Teil 2) \\ 09.09.2018: Rheumatologische \\ Schmerztherapie
}

08.09.-16.12.2018, Hamburg

Rheumatologische Fachassistenz

Grundkurs

Fachliche Leitung:

Dr. med. Florian Schuch

19.09.-22.09.2018

CME: Zertifizierung wird

beantragt

46. Kongress der Deutschen

Gesellschaft für Rheumatologie

Fachliche Leitung:

Prof. Dr. med. Christoph Fiehn

Prof. Dr. med. Bernd Swoboda

\section{Kontakt}

Rheumatologische

Fortbildungsakademie $\mathrm{GmbH}$

Wilhelmine-Gemberg-Weg 6,

Aufgang C, 10179 Berlin

T: $030 / 240484-78$ oder -82

F: $030 / 240484-79$ oder -89

E-Mail: info@rhak.de

www.rheumaakademie.de

Besuchen Sie uns bei Facebook!

\section{WEITERBILDUNGSWOCHENENDEN RHEUMATOLOGIE 2018}

Weitere Informationen zu den Veranstaltungen, das Rheumaakademie Jahresprogramm und die Veranstaltungstermine 2018 finden Sie online unter www.rheumaakademie.de.
25. bis 27. Mai 2018 (Immanuel Krankenhaus, Berlin-Buch) Rheuma und Alter/ Medizinische Begutachtung in der Rheumatologie Teil 1 und Teil 2

7. bis 9. September 2018 (Rheumazentrum Ruhrgebiet, Herne) Kinderwunsch und Schwangerschaft/ Kapillarmikroskopie-Teil 2/ Rheumatologische Schmerztherapie

16. bis 18. November 2018 (Universitätsklinikum Heidelberg) Klinische Immunologie/ Labordiagnostik rheumatischer Krankheiten-Teil 1/ Osteologie

Wir freuen uns auf Ihre Anmeldungen an: info@rheumaakademie.de 